\title{
WATER-QUALITY RECONNAISSANCE AND STREAMFLOW GAIN AND LOSS OF YOCUM CREEK BASIN, CARROLL COUNTY, ARKANSAS
}

by Robert L. Joseph and W. Reed Green

U.S. GEOLOGICAL SURVEY

Open-File Report 94-537

Prepared in cooperation with the ARKANSAS SOIL AND WATER CONSERVATION COMMISSION

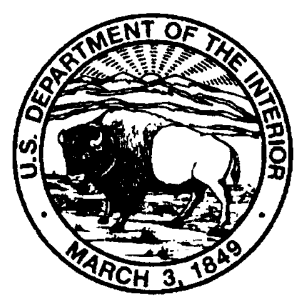




\section{U.S. DEPARTMENT OF THE INTERIOR \\ BRUCE BABBITT, Secretary}

U.S. GEOLOGICAL SURVEY

GORDON P. EATON, Director

For additional information

write to:

District Chief

U.S. Geological Survey, WRD

401 Hardin Road

Little Rock, AR 72211
Copies of this report can be purchased from:

U.S. Geological Survey

Earth Science Information Center

Open-File Reports Section

Box 25286, MS 517

Denver Federal Center

Denver, CO 80225 


\section{CONTENTS}

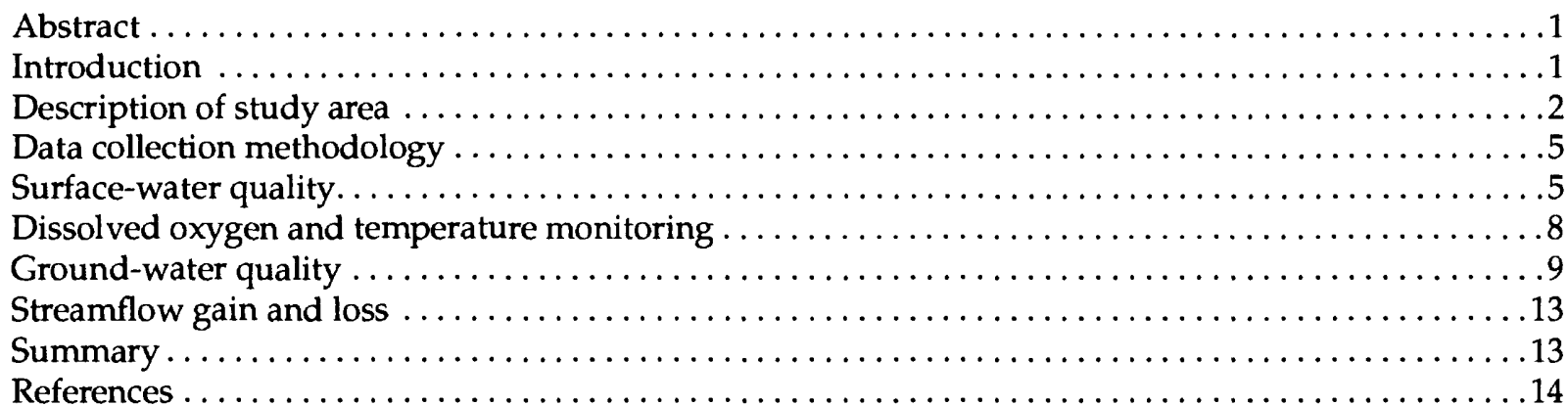

ILLUSTRATIONS

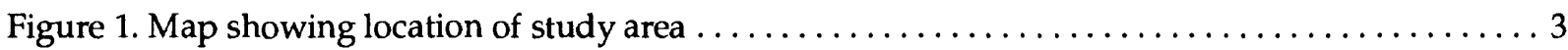

2. Map showing location of sampling sites and gaining and losing stream reaches $\ldots \ldots \ldots \ldots 4$

3. Graph showing comparison of dissolved oxygen concentrations between sites YM-3 and

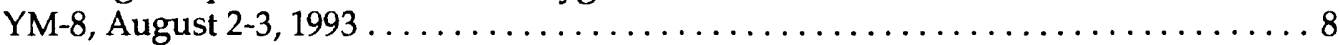

4. Graph showing comparison of temperatures between sites $\mathrm{YM}-3$ and $\mathrm{YM}-8$, August 2-3, 1993.

TABLES

Table 1. Sampling site descriptions of surface-water sites in the Yocum Creek Basin .......... 5

2. Discharge and water-quality data for surface-water sites located in the Yocum Creek

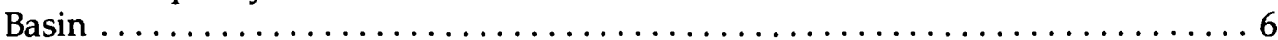

3. Water-quality data for selected surface-water sites, wells, and springs located in the Yocum

Creek Basin. .......................................

4. Sampling site descriptions of wells and springs in the Yocum Creek Basin .......... 10

5 . Well depth and water-quality data for wells located in the Yocum Creek Basin $\ldots \ldots \ldots \ldots 10$

6. Organic compound data for selected wells and springs in the Yocum Creek Basin $\ldots \ldots \ldots 11$

7. Discharge and water-quality data for springs located in the Yocum Creek Basin ........ 12 


\section{CONVERSION FACTORS AND VERTICAL DATUM}

Multiply

By

To obtain

inch (in.)

25.4

foot $(\mathrm{ft})$

0.3048

millimeter

yard (yd)

0.9144

mile (mi)

1.609

meter

square mile $\left(\mathrm{mi}^{2}\right)$

2.590

0.02832

cubic foot per second $\left(\mathrm{ft}^{3} / \mathrm{s}\right)$

0.3048

foot per minute $(\mathrm{ft} / \mathrm{min})$

0.1894

0.063008

meter

foot per mile ( $\mathrm{ft} / \mathrm{mi}$ )

0.063008

kilometer

square kilometer

cubic meter per second

meter per minute

meter per kilometer

liter per second

Temperature in degrees Celsius $\left({ }^{\circ} \mathrm{C}\right)$ can be converted to degrees Fahrenheit $\left({ }^{\circ} \mathrm{F}\right)$ as follows:

$$
{ }^{\circ} \mathrm{F}=1.8^{\circ} \mathrm{C}+32
$$

Sea level: In this report "sea level" refers to the National Geodetic Vertical Datum of 1929--a geodetic datum derived from a general adjustment of the first-order level nets of the United States and Canada, formerly called Sea Level Datum of 1929. 


\title{
WATER-QUALITY RECONNAISSANCE AND STREAMFLOW GAIN AND LOSS OF YOCUM CREEK BASIN, CARROLL COUNTY, ARKANSAS
}

\author{
By Robert L. Joseph and W. Reed Green
}

\begin{abstract}
A study of the Yocum Creek Basin conducted between July 27 and August 3, 1993, described the surface- and ground-water quality of the basin and the streamflow gain and loss. Water samples were collected from 12 sites on the mainstem of Yocum Creek and 2 tributaries during periods of low to moderate streamflow (less than 40 cubic feet per second). Water samples were collected from 5 wells and 12 springs located in the basin.

In 14 surface-water samples, nitrite plus nitrate concentrations ranged from 1.3 to 3.8 milligrams per liter as nitrogen. Orthophosphorus concentrations ranged from 0.01 to 0.06 milligrams per liter as phosphorous. Fecal coliform bacteria counts ranged from 9 to 220 colonies per 100 milliliters, with a median of 49 colonies per 100 milliliters. Fecal streptococci bacteria counts ranged from 37 to 1,500 colonies per 100 milliliters with a median of 420 colonies per 100 milliliters. Analyses for selected metals collected near the mouth of Yocum Creek indicate that metals are not present in significant concentrations in surface-water samples.

Diel dissolved oxygen concentrations and temperatures were measured at two sites on the mainstem of the stream. At the upstream site, dissolved oxygen concentrations ranged from 6.2 to 9.9 milligrams per liter and temperatures ranged from 18.5 to 23.0 degrees Celsius. Dissolved oxygen concentrations were higher and temperature values were lower at the upstream site than those at the downstream site.
\end{abstract}

Five wells were sampled in the basin and dissolved ammonia was present in concentrations ranging from 0.01 to 0.07 milligrams per liter as nitrogen. Dissolved nitrite plus nitrate was present in wells, with concentrations ranging from less than 0.02 to 6.0 milligrams per liter as nitrogen. Volatile organic compound samples were collected at two wells and two springs. Chloroform was the only volatile organic compound found to be above the detection limit. Analysis indicated that 0.2 micrograms per liter of chloroform was present in one spring-water sample.

In springs sampled, nitrite plus nitrate concentrations ranged from 1.4 to 7.0 milligrams per liter as nitrogen. Dissolved ammonia plus organic nitrogen concentrations ranged from less than 0.2 to 0.49 milligrams per liter as nitrogen. Orthophosphorus concentrations ranged from 0.01 to 0.07 milligrams per liter as phosphorus. Fecal coliform bacteria counts ranged from 3 to 200 colonies per 100 milliliters, with a median of 18 colonies per 100 milliliters. Fecal streptococci bacteria counts ranged from 110 to more than 2,000 colonies per 100 milliliters with a median of 350 colonies per 100 milliliters.

Large producing springs located in the mid to upper reaches of the basin contribute most of the flow to Yocum Creek. Streamflow increased an average of 29 percent on the mainstem of the stream. One losing reach was discovered on the mainstem of the stream and two losing reaches on tributaries to the mainstem. Surface flow steadily decreased along these reaches to the point where surface flow was not present, and the streambed became dry. These observations suggest that significant interaction exists between the underlying Springfield aquifer and surface flow in the Yocum Creek Basin.

\section{INTRODUCTION}

The purpose of this report is to describe the current water-quality conditions within the Yocum Creek Basin using physical, nutrient, bacteriological, common constituent, selected metal data, and volatile organic compound data collected at various surface- and ground-water sites in the basin, and to identify gaining and losing streamflow reaches of Yocum Creek and its tributaries. This report was prepared by the U.S. Geological Survey (USGS) in cooperation with the Arkansas Soil and Water Conservation Commission. 
The authors wish to thank the following land owners Mryl Bradley, Quinton Tipton, Terry Benson, Kreglyn Garrett, Kirby Garrett, and Stephen Strafford for granting permission and assistance in sampling of their wells and springs. Additional thanks is extended to Leon Duncan of the Carroll County Cooperative Extension Service.

\section{DESCRIPTION OF STUDY AREA}

Yocum Creek, in northwestern Arkansas, drains an area of $72.8 \mathrm{mi}^{2}$ (Sullavan, 1974), and flows northward through Carroll County (fig. 1) in the Springfield Plateau physiographic section (Fenneman, 1938). The Springfield Plateau topography is characterized by gentle to moderate slopes, ranging from 12 to 50 percent. A large part of this section is dissected by streams that form V-shaped valleys. The study area (fig. 2) has a dendritic drainage pattern with land-surface elevations ranging from approximately $915 \mathrm{ft}$ above sea level near Table Rock Lake to $1,850 \mathrm{ft}$ above sea level in the southem portion of the study area. Yocum Creek has a mean gradient of $18 \mathrm{ft} / \mathrm{mi}$ and a mean channel width of $72 \mathrm{ft}$.

Agricultural development over the last 10 years has resulted in numerous changes in land use, which may affect ground- and surface-water quality. The economic base of Carroll County has undergone changes as well; farming has become more diversified with increased chicken broiler and turkey production over the past decade (Fowlkes and others, 1981). Located in the Yocum Creek Basin are 77 chicken broiler houses and 49 turkey houses, which annually produce an estimated 5 million broilers and 1.75 million turkeys, respectively. Approximately 1.25 million laying hens, 17,000 beef cattle, and 900 dairy cattle are in the Yocum Creek Basin (Leon Duncan, Carroll County Cooperative Extension Service, oral commun., 1994).

Mean annual air temperature in the Yocum Creek Basin is $59^{\circ} \mathrm{F}$, with an average of 52.7 in. of precipitation annually (U.S. Department of Commerce, National Oceanic and Atmospheric Administration, 1992). The normal mean air temperature for the months of May through August (1961-90) is $71^{\circ} \mathrm{F}$, however, in 1993, during this 4month period the mean air temperature was $74^{\circ} \mathrm{F}$. The normal precipitation during the months of May through August is $17.5 \mathrm{in}$., however, 18.9 in. of precipitation were recorded during this period in 1993 (Charles McDonald, National Weather Service, written and oral commun., 1993).

The surficial geology in the headwaters of Yocum Creek consists of Batesville Sandstone of Mississippian age. The Batesville is a coarse- to medium-grained, buff-colored, calcareous sandstone (Croneis, 1930). The average thickness of Batesville Sandstone is approximately $25 \mathrm{ft}$ in the study area. The Batesville Sandstone is composed of sandstone, shale, limestone, and minor amounts of coal, which can be locally fractured with bedding planes that usually yield 2 to $5 \mathrm{gal} / \mathrm{min}$ to wells, except for larger sandstone formations, which may yield $25 \mathrm{gal} / \mathrm{min}$ (Lamonds, 1972).

The largest part of the basin lies on the Boone Formation of Mississippian age. The Boone Formation is normally 300 to $350 \mathrm{ft}$ thick in northern Arkansas and is comprised of limestone, chert, and minor beds of shale and sandstone (Frezon and Glick, 1959). Residual cherty rubble yields 2 to $5 \mathrm{gal} / \mathrm{min}$ to wells, however, many large springs and wells tap large solution channels, which may yield more than $25 \mathrm{gal} / \mathrm{min}$ to wells. The movement of ground water along fractures and bedding planes in this formation produces an abundant number of caves, solution channels, and sinkholes.

The lower part of Yocum Creek, near Table Rock Lake, lies on the Cotter Dolomite of Ordovician age. The Cotter Dolomite consists of a massive, medium-grained, gray dolomitic rock to a fine-grained earthy, white to buff dolomitic rock (Croneis, 1930). The formation consists largely of dolostone, chert, limestone, and smaller amounts of sandstone and shale. The Cotter Dolomite has been reported to be as thick as $500 \mathrm{ft}$ in Boone County directly east of the study area (Caplan, 1960). Fractures in dolomite and dolomitic limestone usually yield 5 to $10 \mathrm{gal} / \mathrm{min}$ to wells. Deeply incised valleys exposed in the Cotter Dolomite commonly produce large springs or seeps. 


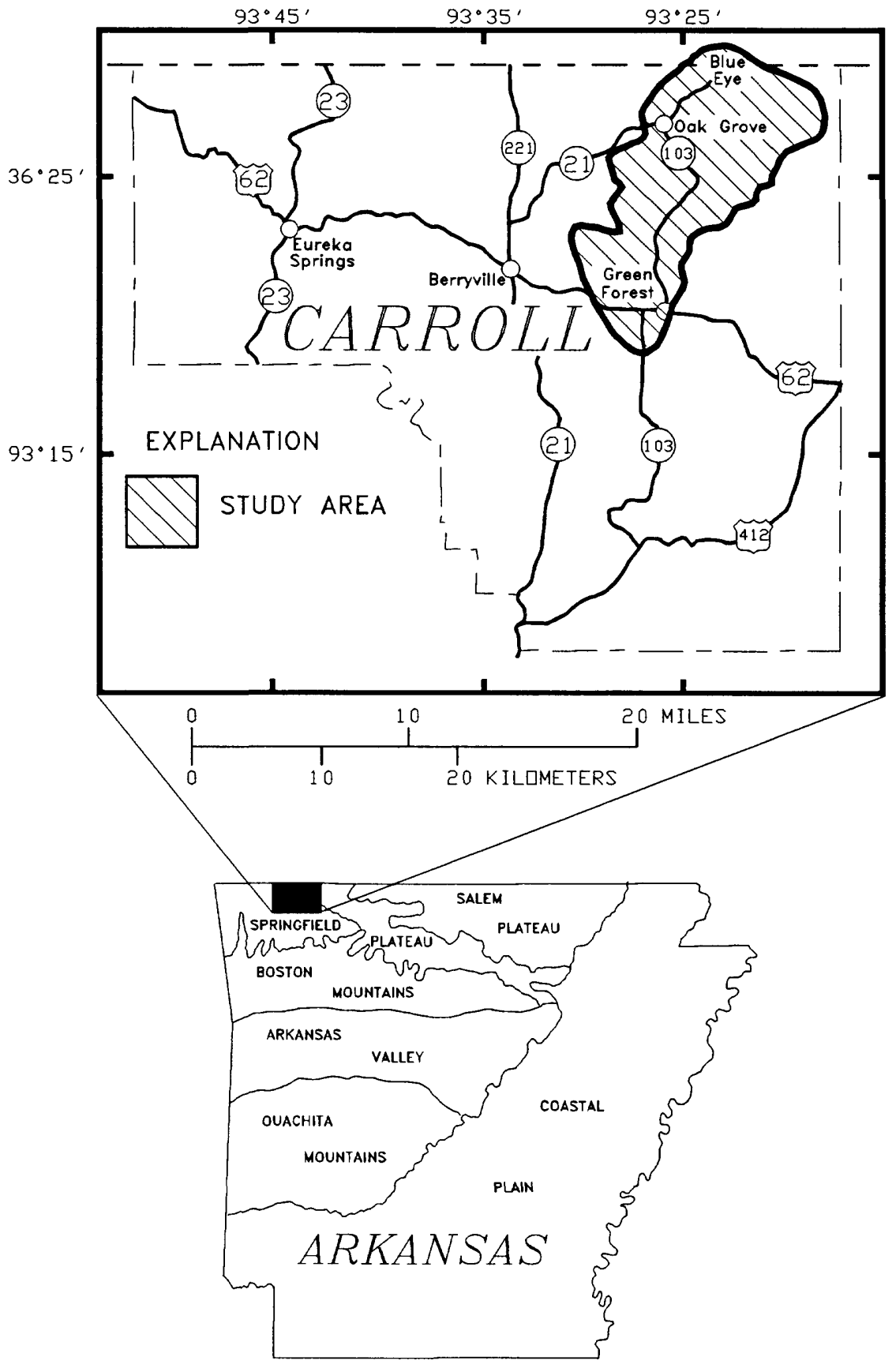

Figure 1.--Location of study area. 


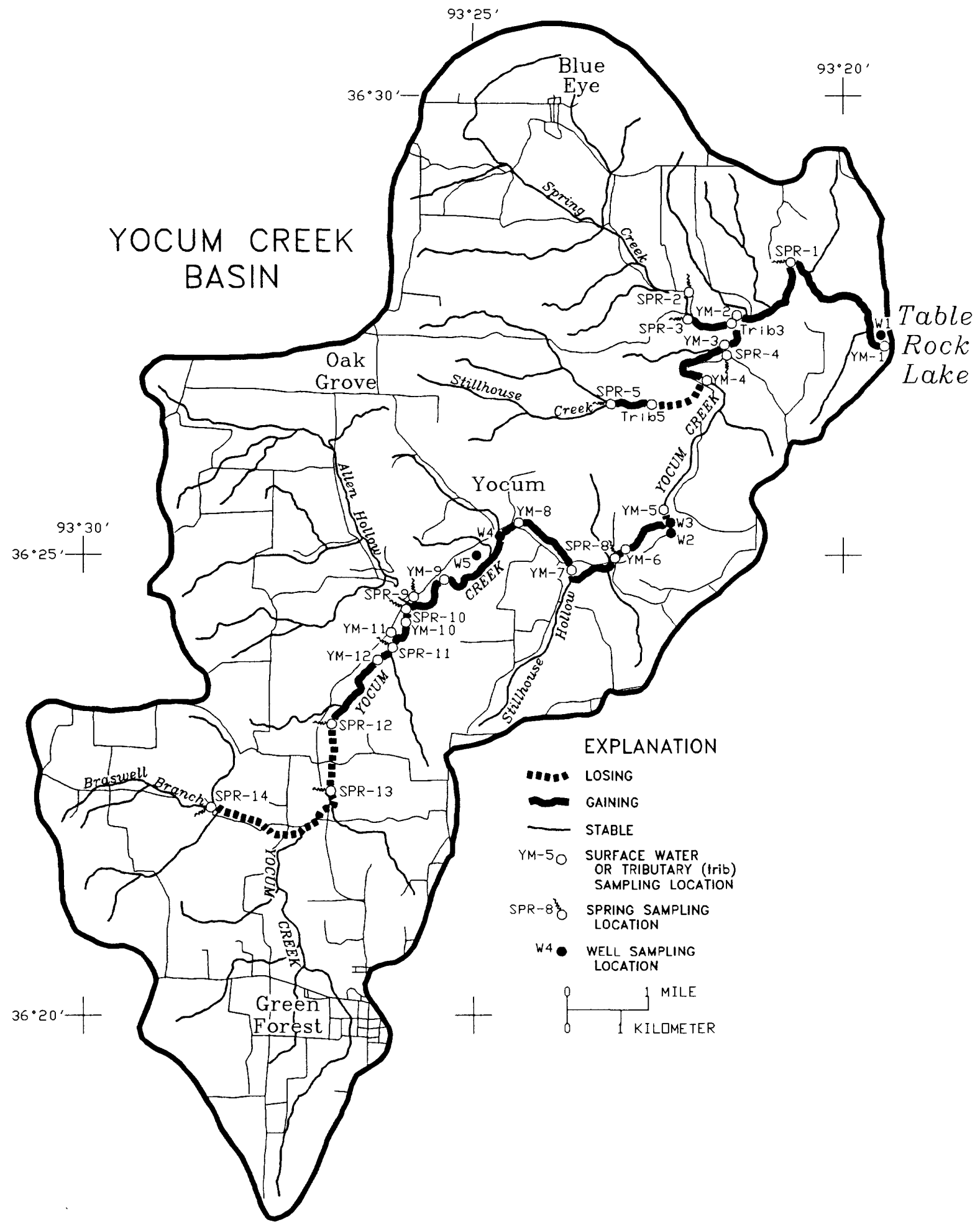

Figure 2.--Location of sampling sites and gaining and losing stream reaches. 


\section{DATA COLLECTION METHODOLOGY}

Ground- and surface-water samples were collected and stream discharge measurements were made within the Yocum Creek Basin between July 27 and August 3, 1993. The sampling site locations were chosen to provide the best understanding of the current water-quality and streamflow conditions. Water samples were collected by hand dipping sample collection bottles into the centroid of flow in the stream and tributaries to the mainstem. Collection, storage, and preservation of samples followed methods outlined by Ward and Harr (1990). Analyses for fecal coliform and fecal streptococcal bacteria were performed following methods described by Britton and Greeson (1987). Discharge was measured at numerous locations on the mainstem of the stream and at tributary inflow points during low-flow conditions (no storm runoff). Discharge measurements were made with a current meter following methods described by Buchanan and Sommers (1984). Comparisons of successive downstream discharge measurements were used to determine if the stream reaches were gaining or losing. Dissolved oxygen concentrations and temperature data were collected for a 24-hour period on an hourly basis at two sites (YM-3 and YM-8) on the mainstem. Water samples from springs were collected by hand dipping sample collection bottles into the orifice of the spring. Water samples from wells were collected from outside faucets prior to any treatment systems.

\section{SURFACE-WATER QUALITY}

Water-quality data were collected at 12 surface-water sites on Yocum Creek and 2 tributaries (table 1) during the study. Specific conductance ranged from 285 to 463 microsiemens per centimeter at 25 degrees Celsius $(\mu \mathrm{S} / \mathrm{cm})$ (table 2). Specific conductance values generally were higher in the headwaters of the basin. Dissolved ammonia concentrations for surface-water sites ranged from 0.01 to 0.03 milligrams per liter $(\mathrm{mg} / \mathrm{L})$ as nitrogen. Dissolved nitrite and dissolved ammonia plus organic nitrogen values were just above detection limits $(0.01 \mathrm{mg} / \mathrm{L})$. Dissolved nitrite plus nitrate concentrations ranged from 1.3 to $3.8 \mathrm{mg} / \mathrm{L}$ as nitrogen. Dissolved orthophosphorus concentrations ranged from 0.01 to $0.06 \mathrm{mg} / \mathrm{L}$ as phosphorus in surface-water sites. Total phosphorous concentrations ranged from less than 0.02 to $0.07 \mathrm{mg} / \mathrm{L}$ as phosphorus in surface-water sites.

Fecal coliform bacteria counts for surface-water sites ranged from 9 to 220 colonies per 100 milliliter (cols $/ 100 \mathrm{~mL}$ ) with a median of $49(\mathrm{cols} / 100 \mathrm{~mL})$. Fecal streptococci bacteria counts for surface-water sites ranged from 37 to $1,500 \mathrm{cols} / 100 \mathrm{~mL}$ with a median of $420 \mathrm{cols} / 100 \mathrm{~mL}$. Analyses for common constituents and selected metals at one surface-water site (YM-1) indicate that most concentrations were below the detections limits (table 3). Calcium, silica, barium, and strontium were measured in low concentrations.

Table 1.--Sampling site descriptions of surface-water sites in the Yocum Creek Basin

[Station number corresponds to national downstream order number assigned by the U.S. Geological Survey]

\begin{tabular}{|c|c|c|c|c|c|}
\hline $\begin{array}{c}\text { Site } \\
\text { number }\end{array}$ & Station number & Station name & Latitude & Longitude & Local site identifier \\
\hline YM-1 & 07053260 & $\begin{array}{l}\text { Yocum Creek at Table Rock Lake near Oak } \\
\text { Grove, Arkansas }\end{array}$ & 362718 & 0931922 & $21 \mathrm{~N} 22 \mathrm{~W} 28 \mathrm{BAD}$ \\
\hline YM-2 & 07053253 & $\begin{array}{l}\text { Yocum Creek upstream from Table Rock } \\
\text { Lake near Oak Grove, Arkansas }\end{array}$ & 362737 & 0932125 & 21N22W19DCB \\
\hline YM-3 & 07053250 & Yocum Creek near Oak Grove, Arkansas & 362714 & 0932123 & $21 \mathrm{~N} 22 \mathrm{~W} 30 \mathrm{ACA}$ \\
\hline YM-4 & 07053247 & $\begin{array}{l}\text { Yocum Creek site } 4 \text { near Oak Grove, } \\
\text { Arkansas }\end{array}$ & 362652 & 0932141 & $21 \mathrm{~N} 22 \mathrm{~W} 30 \mathrm{CAC}$ \\
\hline YM-5 & 07053246 & Yocum Creek northeast of Yocum, Arkansas & 362532 & 0932217 & 20N23W01AAC \\
\hline YM-6 & 07053245 & Yocum Creek east of Yocum, Arkansas & 362501 & 0932244 & 20N23W01CDA \\
\hline YM-7 & 07053243 & Yocum Creek southeast of Yocum, Arkansas & 362451 & 0932331 & 20N23W11ABA \\
\hline YM-8 & 07053242 & Yocum Creek at Yocum, Arkansas & 362518 & 0932416 & $20 \mathrm{~N} 23 \mathrm{~W} 02 \mathrm{CBB}$ \\
\hline YM-9 & 07053239 & Yocum Creek near Yocum, Arkansas & 362440 & 0932513 & 20N23W10BCA \\
\hline YM-10 & 07053236 & $\begin{array}{l}\text { Yocum Creek site } 10 \text { near Green Forest, } \\
\text { Arkansas }\end{array}$ & 362423 & 0932537 & 20N23W04DAB \\
\hline YM-11 & 07053235 & $\begin{array}{l}\text { Yocum Creek site } 11 \text { near Green Forest, } \\
\text { Arkansas }\end{array}$ & 362405 & 0932544 & 20N23WO9DCD \\
\hline YM-12 & 07053234 & $\begin{array}{l}\text { Yocum Creek site } 12 \text { near Green Forest, } \\
\text { Arkansas }\end{array}$ & 362357 & 0932553 & $20 \mathrm{~N} 23 \mathrm{~W} 16 \mathrm{ABB}$ \\
\hline Trib-3 & 07053252 & Spring Creek near Oak Grove, Arkansas & 362734 & 0932126 & 21N22W19DCC \\
\hline Trib-5 & 07053248 & $\begin{array}{l}\text { Stillhouse Hollow Creek near Oak Grove, } \\
\text { Arkansas }\end{array}$ & 362639 & 0932206 & $21 \mathrm{~N} 22 \mathrm{~W} 30 \mathrm{CCC}$ \\
\hline
\end{tabular}




\section{Table 2.--Discharge and water-quality data for surface-water sites located in the Yocum Creek Basin}

[Temperature reported to nearest 0.5 degrees Celsius; ${ }^{\circ} \mathrm{C}$, degrees Celsius; five digit numbers in parentheses are STORET parameter codes used for computer storage of data; $\mathrm{mm}$ of $\mathrm{Hg}$, millimeters of mercury; $\mathrm{tt}^{3} / \mathrm{s}$, cubic feet per second; $\mu \mathrm{S} / \mathrm{cm}$ at $25^{\circ} \mathrm{C}$, microsiemens per centimeter at 25 degrees Celsius; $\mathrm{mg} / \mathrm{L}$, milligrams per liter; $\mathrm{WH}$, whole water; FET, fixed endpoint titration; $F$, field; <, less than; cols/100 mL, number of colonies per 100 milliliters of sample; $K$, non-ideal count]

\begin{tabular}{|c|c|c|c|c|c|c|c|c|c|c|}
\hline Site ID & $\begin{array}{l}\text { Date of } \\
\text { sample }\end{array}$ & $\begin{array}{l}\text { Time of } \\
\text { sample }\end{array}$ & $\begin{array}{l}\text { Water } \\
\text { temperature } \\
\left({ }^{\circ} \mathrm{C}\right) \\
(00010)\end{array}$ & $\begin{array}{c}\text { Air pressure } \\
(\mathrm{mm} \text { of } \mathrm{Hg}) \\
(00025)\end{array}$ & $\begin{array}{c}\text { Discharge, } \\
\text { instan- } \\
\text { taneous } \\
\left(\mathrm{ft}^{3} / \mathrm{s}\right) \\
(00061)\end{array}$ & \multicolumn{2}{|c|}{$\begin{array}{c}\text { Specific } \\
\text { conductance } \\
(\mu \mathrm{S} / \mathrm{cm} \text { at } \\
\left.25^{\circ} \mathrm{C}\right) \\
(00095)\end{array}$} & $\begin{array}{c}\text { Oxygen, } \\
\text { dissolved } \\
(\mathrm{mg} / \mathrm{L}) \\
(00300)\end{array}$ & $\begin{array}{c}\mathrm{pH}, \\
\text { field } \\
\text { (standard } \\
\text { units) } \\
(00400)\end{array}$ & $\begin{array}{l}\text { Alkalinity, } \\
\text { WH, FET, F } \\
(\mathrm{mg} / \mathrm{L} \text { as } \\
\left.\mathrm{CaCO}_{3}\right) \\
(00410)\end{array}$ \\
\hline$\overline{Y M}-1$ & $7-27-93$ & 1240 & 24.0 & 744 & 40 & \multicolumn{2}{|c|}{310} & 9.1 & 8.0 & 136 \\
\hline YM-2 & $7-27-93$ & 1805 & 23.5 & 732 & 35 & \multicolumn{2}{|c|}{308} & 9.0 & 8.3 & 138 \\
\hline YM-3 & $7-28-93$ & 0830 & 22.0 & 736 & 24 & \multicolumn{2}{|c|}{335} & 7.6 & 7.8 & 162 \\
\hline YM-4 & $7-28-93$ & 1245 & 25.0 & 735 & 20 & \multicolumn{2}{|c|}{316} & 9.6 & 8.3 & 142 \\
\hline YM-5 & $7-28-93$ & 1530 & 25.0 & 734 & 22 & \multicolumn{2}{|c|}{463} & 7.7 & 7.8 & 138 \\
\hline YM-6 & $7-29-93$ & 0850 & 19.5 & 737 & 20 & \multicolumn{2}{|c|}{356} & 8.3 & 7.5 & 148 \\
\hline YM-7 & $7-29-93$ & 1010 & 20.0 & 737 & 15 & \multicolumn{2}{|c|}{348} & 8.7 & 7.9 & 156 \\
\hline YM-8 & $7-29-93$ & 1210 & 21.0 & 736 & 14 & \multicolumn{2}{|c|}{339} & 10.0 & 8.0 & 142 \\
\hline YM-9 & $7-29-93$ & 1540 & 21.5 & 733 & 14 & \multicolumn{2}{|c|}{345} & 9.5 & 7.9 & 144 \\
\hline YM-10 & $8-02-93$ & 1300 & 19.0 & 732 & 12 & \multicolumn{2}{|c|}{352} & 9.0 & 7.1 & 160 \\
\hline YM-11 & $8-02-93$ & 1425 & 24.0 & 732 & 5.0 & \multicolumn{2}{|c|}{399} & 8.8 & 7.6 & 154 \\
\hline YM-12 & $8-02-93$ & 1400 & 24.5 & 732 & 3.5 & \multicolumn{2}{|c|}{388} & 9.2 & 7.8 & 154 \\
\hline Trib-3 & $7-27-93$ & 1740 & 18.0 & 732 & 8.6 & 2 & & 9.1 & 8.8 & 144 \\
\hline Trib-5 & $7-28-93$ & 1330 & 19.0 & 734 & 2.6 & 3. & & 8.8 & 7.4 & 156 \\
\hline Minimum & & & 18.0 & & 2.6 & & & 7.6 & 7.1 & 136 \\
\hline Maximum & & & 25.0 & & 40 & & & 10.0 & 8.8 & 162 \\
\hline Median & & & 22.0 & & 15 & & & 9.0 & 7.8 & 146 \\
\hline Site ID & $\begin{array}{c}\text { Nitrogen } \\
\text { ammonia, } \\
\text { dissolved } \\
(\mathrm{mg} / \mathrm{L} \text { as } \mathrm{N}) \\
(00608)\end{array}$ & $\begin{array}{c}\text { Nitrogen, } \\
\text { nitrite } \\
\text { dissolved } \\
(\mathrm{mg} / \mathrm{L} \text { as } \mathrm{N}) \\
(00613)\end{array}$ & $\begin{array}{l}\text { Nitrogen, } \\
\text { ammonia plus } \\
\text { organic } \\
\text { dissolved } \\
(\mathrm{mg} / \mathrm{L} \text { as } \mathrm{N}) \\
(00623\end{array}$ & $\begin{array}{r}\text { Nitrog } \\
\text { nitrite } \\
\text { nitra } \\
\text { dissol } \\
(\mathrm{mg} / \mathrm{L} \mathrm{a} \\
(0063\end{array}$ & $\begin{array}{l}\text { n, } \\
\text { lus } \\
\text { ed } \\
\text { N) }\end{array}$ & $\begin{array}{l}\text { lonus, } \\
\text { al } \\
\text { as P) } \\
65 \text { ) }\end{array}$ & $\begin{array}{r}\text { Phosp } \\
\text { Ort } \\
\text { disso } \\
(\mathrm{mg} / \mathrm{L} \\
(006\end{array}$ & $\begin{array}{l}\text { lonus } \\
\text { lo, } \\
\text { lved } \\
\text { as) } \\
71 \text { ) }\end{array}$ & $\begin{array}{c}\text { Coliform } \\
\text { fecal, } \\
0.7 \text { micron } \\
\text { membrane } \\
\text { filter } \\
\text { (cols } / 100 \mathrm{~mL}) \\
(31625)\end{array}$ & $\begin{array}{c}\text { Strepto- } \\
\text { cocci } \\
\text { fecal, } \\
\text { KF agar } \\
\text { (cols/100 mL) } \\
(31673)\end{array}$ \\
\hline$\overline{\mathrm{YM}-1}$ & 0.01 & 0.01 & $<0.20$ & 1.9 & & 0.03 & & 03 & K55 & $\mathrm{K} 37$ \\
\hline YM-2 & .03 & .01 & .39 & 2.0 & & .03 & & 03 & 200 & 160 \\
\hline YM-3 & .02 & .01 & $<.20$ & 2.3 & & .05 & & 03 & K42 & $\mathrm{K} 320$ \\
\hline YM-4 & .02 & .01 & .24 & 2.4 & & .07 & & 04 & K45 & 420 \\
\hline YM-5 & .02 & .01 & $<.20$ & 2.5 & & .07 & & 04 & $\mathrm{~K} 27$ & 440 \\
\hline YM-6 & .02 & .01 & .28 & 2.7 & & .03 & & 03 & K70 & 860 \\
\hline YM-7 & .02 & .01 & $<.20$ & 2.9 & & .04 & & 04 & 87 & 780 \\
\hline YM-8 & .02 & .01 & $<.20$ & 2.5 & & .04 & & 04 & K58 & 1,100 \\
\hline YM-9 & .02 & .02 & $<.20$ & 3.8 & & .04 & & 34 & $\mathrm{~K} 42$ & 390 \\
\hline YM-10 & .03 & $<.01$ & $<.20$ & 2.7 & & .03 & & 04 & $\mathrm{~K} 52$ & 520 \\
\hline YM-11 & .03 & $<.01$ & $<.20$ & 3.6 & & .05 & & 66 & K24 & 720 \\
\hline YM-12 & .03 & $<.01$ & $<.20$ & 3.1 & & .05 & & 06 & $\mathrm{~K} 12$ & 1,500 \\
\hline Trib-3 & .01 & .01 & $<.20$ & 1.8 & & .02 & & 02 & $\mathrm{~K} 220$ & 340 \\
\hline Trib-5 & .01 & .01 & $<.20$ & 1.3 & & $<.02$ & & 1 & K9 & 140 \\
\hline Minimum & .01 & $<.01$ & $<.20$ & 1.3 & &. .02 & & 1 & K9 & $\mathrm{K} 37$ \\
\hline Maximum & .03 & .02 & .39 & 3.8 & & .07 & & 06 & $\mathrm{~K} 220$ & 1,500 \\
\hline Median & .02 & .01 & $<.20$ & 2.5 & & .04 & & 04 & 49 & 420 \\
\hline
\end{tabular}




\section{Table 3.--Water-quality data for selected surface-water sites, wells, and springs located in the Yocum Creek Basin}

$\left[\mu \mathrm{S} / \mathrm{cm}\right.$ at $25^{\circ} \mathrm{C}$, microsiemens per centimeter at 25 degrees Celsius; five digit numbers in parentheses are STORET parameter codes used for computer storage of data; $\mathrm{mg} / \mathrm{L}$, milligrams per liter; $<$, less than; $\mu g / \mathrm{L}$, micrograms per liter; ${ }^{\circ} \mathrm{C}$, degrees Ceisius]

\begin{tabular}{|c|c|c|c|c|c|c|c|c|c|c|c|c|c|}
\hline Site ID & $\begin{array}{l}\text { Date of } \\
\text { sample }\end{array}$ & $\begin{array}{l}\text { Time of } \\
\text { sample }\end{array}$ & & $\begin{array}{l}\text { ecific } \\
\text { duc- } \\
\text { nce } \\
/ \mathrm{cm} \text { at } \\
\left.{ }^{\circ} \mathrm{C}\right) \\
095)\end{array}$ & & $\begin{array}{l}\text { H, } \\
\text { b } \\
\text { 103) }\end{array}$ & $\begin{array}{r}\text { Hard } \\
\text { to } \\
(\mathrm{mg} \\
\mathrm{Ca} \\
(009\end{array}$ & & $\begin{array}{c}\begin{array}{c}\text { Noncar- } \\
\text { bonate, } \\
\text { hardness }\end{array} \\
(\mathrm{mg} / \mathrm{L} \text { as } \\
\left.\mathrm{CaCO}_{3}\right) \\
(00902)\end{array}$ & $\begin{array}{c}\text { Calcium, } \\
\text { dissolved } \\
(\mathrm{mg} / \mathrm{L} \text { as } \\
\mathrm{Ca}) \\
(00915)\end{array}$ & $\begin{array}{c}\text { Magne- } \\
\text { sium, } \\
\text { dissolved } \\
(\mathrm{mg} / \mathrm{L} \text { as } \\
\mathrm{mg}) \\
(00925)\end{array}$ & $\begin{array}{c}\text { Sodium, } \\
\text { dissolved } \\
\text { (mg/L as } \\
\mathrm{Na}) \\
(00930)\end{array}$ & $\begin{array}{l}\text { Sodium, } \\
\text { adsorp- } \\
\text { tion } \\
\text { rate } \\
\text { (ratio) } \\
\text { (00931) }\end{array}$ \\
\hline$\overline{Y M-1}$ & $7-27-93$ & 1240 & & 310 & & 2 & 150 & & 12 & 55 & 2.6 & 3.2 & 0.1 \\
\hline W1 & $7-27-93$ & 1700 & & 140 & & 9 & 240 & & 29 & 50 & 27 & 2.8 & 0 \\
\hline W5 & $7-29-93$ & 1500 & & 66 & & 5 & 190 & & 33 & 75 & 1.4 & 1.8 & 0 \\
\hline SPR-3 & $7-28-93$ & 1040 & & 288 & & 4 & 140 & & 7 & 52 & 1.3 & 3.0 & .1 \\
\hline SPR-13 & $8-02-93$ & 1615 & & 160 & & 2 & 150 & & 0 & 58 & 1.8 & 3.7 & .1 \\
\hline Site ID & $\begin{array}{c}\text { Sodium } \\
\text { percent } \\
\text { (percent) } \\
(00932)\end{array}$ & $\begin{array}{c}\begin{array}{c}\text { Potas- } \\
\text { sium, } \\
\text { dissolved } \\
(\mathrm{mg} / \mathrm{L} \\
\text { as } \mathrm{K}) \\
(00935)\end{array}\end{array}$ & & $\begin{array}{l}\text { ride, } \\
\text { olved } \\
L \text { as } \\
\text { 1) } \\
40)\end{array}$ & & $\begin{array}{l}\text { ate, } \\
\text { lved } \\
\text { L as } \\
4 \text { ) } \\
45)\end{array}$ & $\begin{array}{r}\text { Flu } \\
\text { dis } \\
(\mathrm{mg}) \\
(0 \times\end{array}$ & $\begin{array}{l}\text { ide, } \\
\text { lved } \\
\text { as F) } \\
50 \text { ) }\end{array}$ & $\begin{array}{c}\text { Silica, } \\
\text { dissolved } \\
(\mathrm{mg} / \mathrm{L} \text { as } \\
\left.\mathrm{SiO}_{2}\right) \\
(00955)\end{array}$ & $\begin{array}{c}\text { Arsenic, } \\
\text { dissolved } \\
(\mu \mathrm{g} / \mathrm{L} \text { as } \\
\text { As }) \\
(01000)\end{array}$ & $\begin{array}{c}\text { Barium, } \\
\text { dissolved } \\
(\mu \mathrm{g} / \mathrm{L} \text { as } \\
\mathrm{Ba}) \\
(01005)\end{array}$ & $\begin{array}{c}\text { Beryl- } \\
\text { lium, } \\
\text { dissolved } \\
(\mu \mathrm{g} / \mathrm{L} \text { as } \\
\mathrm{Be}) \\
(01010)\end{array}$ & $\begin{array}{c}\text { Boron, } \\
\text { dissolved } \\
(\mu \mathrm{g} / \mathrm{L} \\
\text { as } \mathrm{B}) \\
(01020)\end{array}$ \\
\hline$\overline{\mathrm{YM}-1}$ & 5 & 2.2 & & & & & & & 11 & $<1$ & 46 & $<1$ & $<20$ \\
\hline W1 & 3 & 4.1 & & & 1 & & & & 9.3 & $<1$ & 8 & $<1$ & 30 \\
\hline W5 & 2 & .82 & & & & & & & 12 & $<1$ & 31 & $<1$ & $<20$ \\
\hline SPR-3 & 5 & 1.7 & & & & & & & 11 & $<1$ & 40 & $<1$ & $<20$ \\
\hline SPR-13 & 5 & 1.1 & & & & & & & 10 & $<1$ & 37 & $<1$ & $<20$ \\
\hline Site ID & $\begin{array}{c}\text { Cadmium, } \\
\text { dissolved } \\
(\mu \mathrm{g} / \mathrm{L} \text { as } \\
\mathrm{Cd}) \\
(01025)\end{array}$ & $\begin{array}{r}\text { Chrom } \\
\text { dissol } \\
(\mu \mathrm{g} / \mathrm{L} \\
\mathrm{Cr} \\
(010\end{array}$ & & $\begin{array}{r}\text { Col } \\
\text { diss } \\
(\mu \mathrm{g}) \\
\mathrm{C} \\
(01\end{array}$ & & $\begin{array}{r}\mathrm{Co} \\
\text { diss } \\
(\mu \mathrm{g} \\
\mathrm{C} \\
(01\end{array}$ & $\begin{array}{l}\text { oper, } \\
\text { olved } \\
\text { L as } \\
\text { u) } \\
040 \text { ) }\end{array}$ & $\begin{array}{r}\text { Ir } \\
\text { diss } \\
(\mu \mathrm{g} \\
\mathrm{F} \\
(01\end{array}$ & $\begin{array}{l}\text { ron, } \\
\text { olved } \\
\text { L as } \\
\text { Fe) } \\
046)\end{array}$ & $\begin{array}{l}\text { Lead, } \\
\text { lissolved } \\
(\mu \mathrm{g} / \mathrm{L} \text { as } \\
\mathrm{Pb}) \\
(01049)\end{array}$ & $\begin{array}{l}\text { Manga- } \\
\text { nese, } \\
\text { issolved } \\
(\mu \mathrm{g} / \mathrm{L} \text { as } \\
\mathrm{Mn}) \\
(01056)\end{array}$ & $\begin{array}{l}\text { Molyb- } \\
\text { denum, } \\
\text { dissolved } \\
(\mu \mathrm{g} / \mathrm{L} \text { as } \\
\text { Mo }) \\
(01060)\end{array}$ & $\begin{array}{c}\text { Nickel, } \\
\text { dissolved } \\
(\mu \mathrm{g} / \mathrm{L} \text { as } \\
\mathrm{Ni}) \\
(01065)\end{array}$ \\
\hline YM-1 & $<1.0$ & & & & & & 10 & & $<3$ & $<10$ & 1.0 & $<10$ & $<10$ \\
\hline W1 & $<1.0$ & & & & & & 10 & & $<3$ & $<10$ & 2.0 & $<10$ & $<10$ \\
\hline W5 & 2.0 & & & & & & 10 & & $<3$ & $<10$ & $<1.0$ & $<10$ & $<10$ \\
\hline SPR-3 & $<1.0$ & & & & & & 10 & & $<3$ & $<10$ & $<1.0$ & $<10$ & $<10$ \\
\hline SPR-13 & 2.0 & & & & & & 10 & & $<3$ & $<10$ & $<1.0$ & $<10$ & $<10$ \\
\hline
\end{tabular}




\section{DISSOLVED OXYGEN AND TEMPERATURE MONITORING}

Hourly dissolved oxygen and temperature values were collected at two sites (YM-3 and YM-8) on the mainstem of Yocum Creek from August 2 to August 3 (figs. 3 and 4). These data were collected to determine the diel fluctuations in dissolved oxygen concentrations and temperature during changes in air temperature and sunlight intensity.

At the downstream site (YM-3), dissolved oxygen concentrations ranged from 5.7 to $9.3 \mathrm{mg} / \mathrm{L}$ with a median of $6.4 \mathrm{mg} / \mathrm{L}$. The minimum dissolved oxygen concentration of $5.7 \mathrm{mg} / \mathrm{L}$ was recorded at 0405 hours and the maximum dissolved oxygen concentration value of $9.3 \mathrm{mg} / \mathrm{L}$ was recorded at 1605 hours. The minimum stream temperature of $21.5^{\circ} \mathrm{C}$ was recorded at 0605 hours, and the maximum stream temperature of $25^{\circ} \mathrm{C}$ was recorded at 1605 hours (fig. 4). The median stream temperature at site $\mathrm{YM}-3$ was $22.8^{\circ} \mathrm{C}$.

At the upstream site (YM-8), dissolved oxygen concentrations ranged from 6.2 to $9.9 \mathrm{mg} / \mathrm{L}$ with a median of $7.8 \mathrm{mg} / \mathrm{L}$. The minimum dissolved oxygen concentration of $6.2 \mathrm{mg} / \mathrm{L}$ was recorded at 0145 hours and the maximum dissolved oxygen concentration of $9.9 \mathrm{mg} / \mathrm{L}$ was recorded at 1345 hours. The minimum stream temperature of 18.5 ${ }^{\circ} \mathrm{C}$ was recorded at 0545 hours and the maximum stream temperature of $23.0^{\circ} \mathrm{C}$ was recorded at 1645 hours. The median stream temperature at site YM-8 was $20.0^{\circ} \mathrm{C}$.

The stream temperature at the downstream site (YM-3) was about 2 degrees warmer than the upstream site (YM-8), which is located closer to the large producing springs in the mid to upper reaches of the basin. The cooler site, YM-8, normally maintained a higher dissolved oxygen concentration than site YM-3.

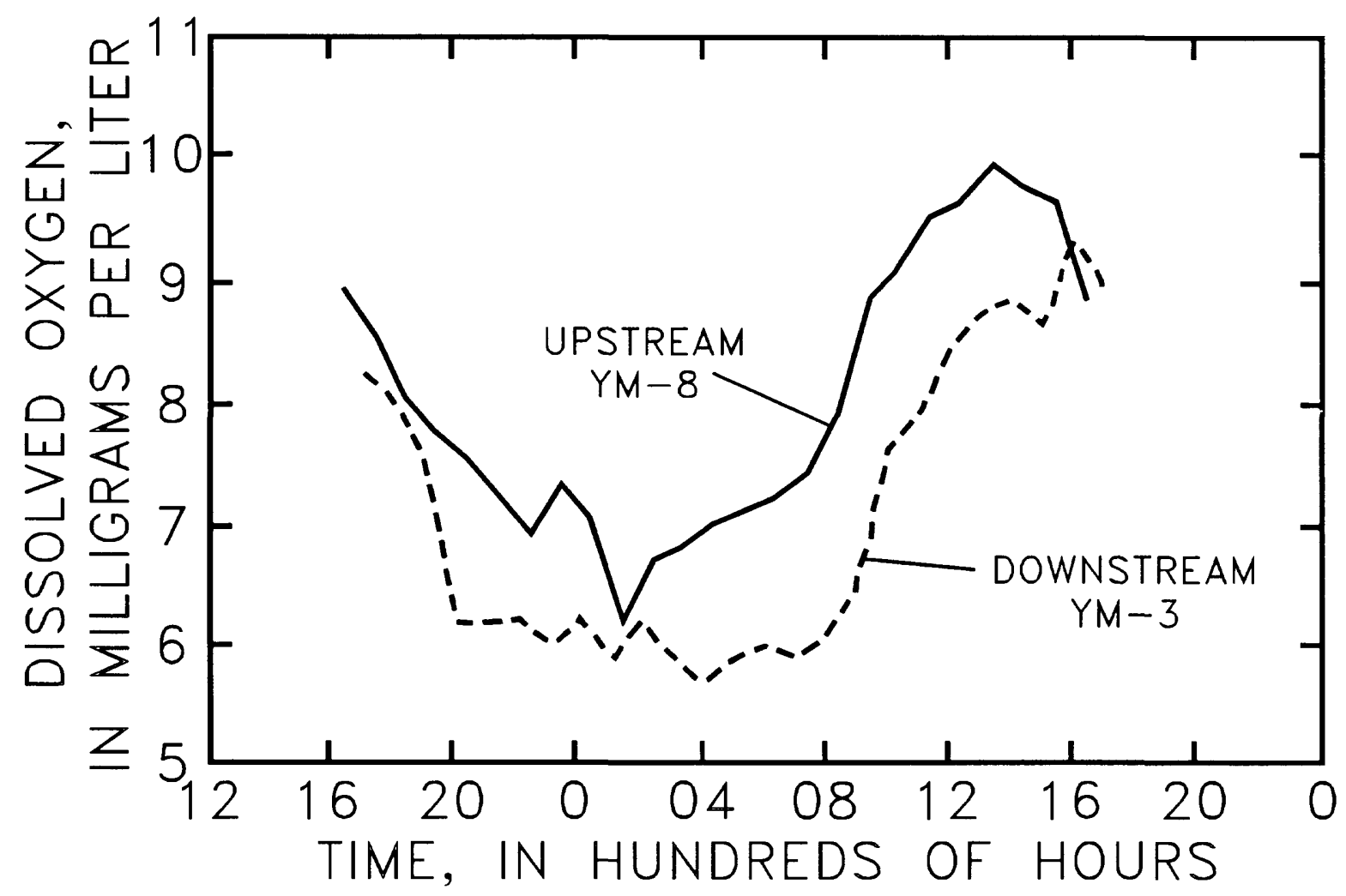

Figure 3.--Comparison of dissolved oxygen concentrations between sites $Y M-3$ and $Y M-8$, August 2-3, 1993. 


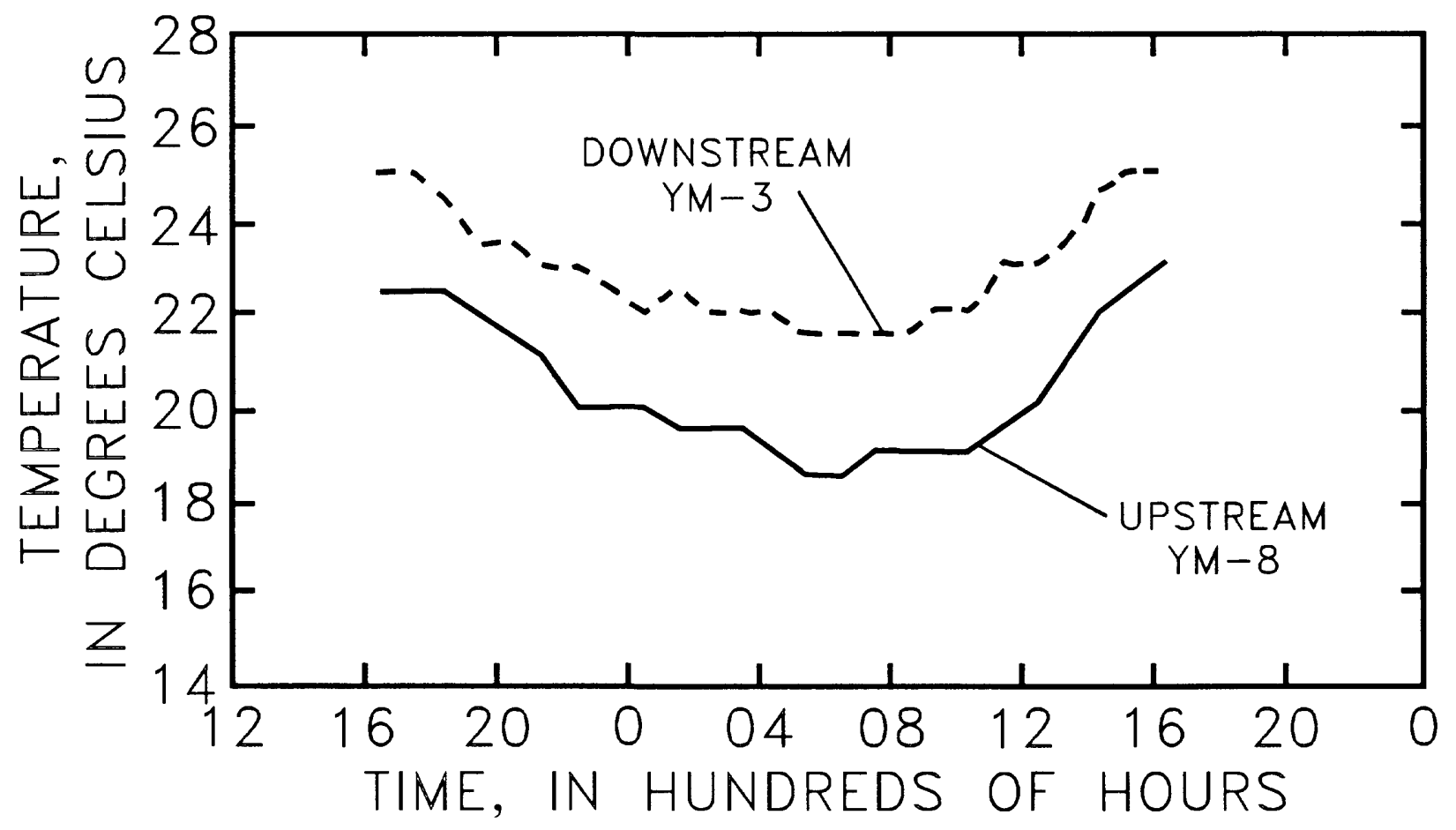

\author{
Figure 4.--Comparison of temperatures between sites \\ YM-3 and YM-8, August 2-3, 1993.
}

\title{
GROUND-WATER QUALITY
}

Water-quality data were collected from five wells in the Yocum Creek Basin (table 4). Three of these wells are considered shallow wells ( 30 to $68 \mathrm{ft}$ ) penetrating the Springfield aquifer and two wells are considered deep ( 350 and $550 \mathrm{ft}$ ) penetrating the Ozark aquifer. Specific conductance values ranged from 361 to $549 \mu \mathrm{S} / \mathrm{cm}$ (table 5). Dissolved ammonia was present in small concentrations in all five wells, and ranged from 0.01 to $0.07 \mathrm{mg} / \mathrm{L}$ as nitrogen. Dissolved nitrite plus nitrate ranged from less than 0.02 to $6.0 \mathrm{mg} / \mathrm{L}$. The sample from well W-4 recorded a dissolved nitrite plus nitrate concentration of $6.0 \mathrm{mg} / \mathrm{L}$ as nitrogen, which is approaching the U.S. Environmental Protection Agency maximum contaminant level for drinking water of $10 \mathrm{mg} / \mathrm{L}$ as nitrogen (U.S. Environmental Protection Agency, 1994). Fecal coliform bacteria counts for wells ranged from less than 3 to 15 cols/100 mL with a median of $3 \mathrm{cols} / 100 \mathrm{~mL}$. Fecal streptococci bacteria counts ranged from less than 4 to $77 \mathrm{cols} / 100 \mathrm{~mL}$ with a median of 17 cols $/ 100 \mathrm{~mL}$. Analyses for common constituents and selected trace metals in wells W1 and W5 indicate that drinking water standards were not exceeded. Volatile organic compound analyses (table 6) indicate that organic compounds were not present above the detection limit.

In springs discharging from the Springfield aquifer, nitrite plus nitrate concentrations ranged from 1.4 to 7.0 $\mathrm{mg} / \mathrm{L}$ as nitrogen (table 7). Dissolved ammonia plus organic nitrogen concentrations ranged from less than 0.02 to $0.49 \mathrm{mg} / \mathrm{L}$ as nitrogen. Dissolved orthophosphorus concentrations ranged from 0.01 to $0.07 \mathrm{mg} / \mathrm{L}$ as phosphorus. Fecal coliform bacteria counts ranged from 3 to $200 \mathrm{cols} / 100 \mathrm{~mL}$, with a median of $18 \mathrm{cols} / 100 \mathrm{~mL}$. Fecal streptococci bacteria counts ranged from 110 to more than $2,000 \mathrm{cols} / 100 \mathrm{~mL}$ with a median of $350 \mathrm{cols} / 100 \mathrm{~mL}$. Analyses for common constituents and selected trace metals in two springs (SPR-3 and SPR-13) indicate that trace metals were present in low concentrations. Volatile organic compound samples were collected at two springs, SPR-3 and SPR-13 (table 8). Chloroform was the only volatile organic compound found to be above the detection limit. Analyses indicated that $0.2 \mu \mathrm{g} / \mathrm{L}$ of chloroform were present in SPR-13. 
Table 4.--Sampling site descriptions of wells and springs in the Yocum Creek Basin

\begin{tabular}{llccc}
\hline Site number & Station name & Latitude & Longitude & Local site identifier \\
\hline W1 & Well 1 & 362724 & 0931857 & 21N22W28AAB \\
W2 & Well 2 & 362518 & 0932213 & 20N22W01DAA \\
W3 & Well 3 & 362521 & 0932213 & 20N23W01ADD \\
W4 & Well 4 & 362510 & 0932433 & 20N23W03DAC \\
W5 & Well 5 & 362502 & 0932447 & 20N23W03DCB \\
SPR-1 & Spring 1 & 362815 & 0932040 & 21N22W20BAB \\
SPR-2 & Spring 2 & 362742 & 0932204 & 21N22W19CBB \\
SPR-3 & Spring 3 & 362735 & 0932159 & 21N22W19CCA \\
SPR-4 & Spring 4 & 362714 & 0932120 & 21N22W30ACA \\
SPR-5 & Spring 5 & 362639 & 0932247 & 21N23W25CDD \\
SPR-8 & Spring 8 & 362501 & 0932253 & 20N23W01CDB \\
SPR-9 & Spring 9 & 362438 & 0932526 & 20N23W09ADA \\
SPR-10 & Spring 10 & 362420 & 0932543 & 20N23W09DBD \\
SPR-11 & Spring 11 & 362403 & 0932545 & 20N23W16ABA \\
SPR-12 & Spring 12 & 362255 & 0932640 & 20N23W20ADB \\
SPR-13 & Spring 13 & 362232 & 0932646 & 20N23W20DCA \\
SPR-14 & Spring 14 & 362210 & 0932821 & 20N24W30BBD \\
\hline
\end{tabular}

Table 5.--Well depth and water-quality data for wells located in the Yocum Creek Basin

[Temperature reported to nearest 0.5 degrees Celsius; ${ }^{\circ} \mathrm{C}$, degrees Celsius; five digit numbers in parentheses are STORET parameter codes used for computer storage of data; $\mu \mathrm{S} / \mathrm{cm}$ at $25^{\circ} \mathrm{C}$, microsiemens per centimeter at 25 degrees Celsius; WH, whole water; FET, fixed endpoint titration; F, field; $\mathrm{mg} / \mathrm{L}$, milligrams per liter; <, less than; cols/100 mL, number of colonies per 100 milliliters of sample; $K$, non-ideal count]

\begin{tabular}{|c|c|c|c|c|c|c|c|c|c|}
\hline Site ID & $\begin{array}{l}\text { Date of } \\
\text { sample }\end{array}$ & $\begin{array}{l}\text { Time of } \\
\text { sample }\end{array}$ & $\begin{array}{c}\text { Water } \\
\text { temper- } \\
\text { ature } \\
\left({ }^{\circ} \mathrm{C}\right)\end{array}$ & $\begin{array}{c}\text { Depth of } \\
\text { well, } \\
\text { total } \\
\text { (feet) } \\
(00010)\end{array}$ & 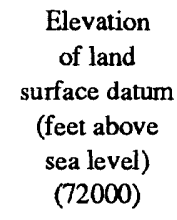 & $\begin{array}{c}\text { Specific } \\
\text { conductance } \\
(\mu \mathrm{S} / \mathrm{cm} \text { at } \\
\left.25^{\circ} \mathrm{C}\right) \\
(00095)\end{array}$ & $\begin{array}{c}\mathrm{pH}, \\
\text { field } \\
\text { (standard } \\
\text { units) } \\
(00400)\end{array}$ & $\begin{array}{l}\text { Alkalinity, } \\
\text { WH, FET, F } \\
(\mathrm{mg} / \mathrm{L} \text { as } \\
\left.\mathrm{CaCO}_{3}\right) \\
(00410)\end{array}$ & $\begin{array}{c}\text { Nitrogen } \\
\text { ammonia, } \\
\text { dissolved } \\
(\mathrm{mg} / \mathrm{L} \text { as } \mathrm{N}) \\
(00608)\end{array}$ \\
\hline$\overline{\mathrm{W} 1}$ & $7-27-93$ & 1700 & 21.0 & 550 & 1,000 & 440 & 7.6 & 208 & 0.02 \\
\hline W2 & $7-28-93$ & 1610 & 21.0 & 40 & 1,080 & 549 & 7.2 & 156 & .01 \\
\hline W3 & $7-28-93$ & 1620 & 16.5 & 350 & 1,080 & 361 & 7.7 & 174 & .07 \\
\hline W4 & $7-29-93$ & 1130 & 17.0 & 30 & 1,100 & 375 & 7.2 & 196 & .02 \\
\hline W5 & $7-29-93$ & 1500 & 16.0 & 68 & 1,110 & 366 & 7.1 & 160 & .01 \\
\hline Minimum & & & 16.0 & 30 & 1,000 & 361 & 7.1 & 156 & .01 \\
\hline Maximum & & & 21.0 & 550 & 1,110 & 549 & 7.7 & 208 & .07 \\
\hline Median & & & 17.0 & 68 & 1,080 & 375 & 7.2 & 174 & .02 \\
\hline Site ID & $\begin{array}{c}\text { Nitrogen, } \\
\text { nitrite } \\
\text { dissolved } \\
(\mathrm{mg} / \mathrm{L} \text { as } \mathrm{N}) \\
(00613)\end{array}$ & $\begin{array}{r}\mathrm{Ni} \\
\text { amm } \\
0 \\
\text { dis } \\
(\mathrm{mg} \\
(0\end{array}$ & $\begin{array}{l}\text { gen, } \\
\text { ia plus } \\
\text { anic } \\
\text { lved } \\
\text { as N) } \\
523 \text { ) }\end{array}$ & $\begin{array}{c}\text { Nitrogen, } \\
\text { nitrite plus } \\
\text { nitrate } \\
\text { dissolved } \\
(\mathrm{mg} / \mathrm{L} \text { as } \mathrm{N}) \\
(00631)\end{array}$ & $\begin{array}{l}\text { Phos- } \\
\text { phorus, } \\
\text { total } \\
(\mathrm{mg} / \mathrm{L} \text { as } \mathrm{P}) \\
(00665)\end{array}$ & $\begin{array}{l}\text { Phos- } \\
\text { phorus } \\
\text { ortho, } \\
\text { dissolved } \\
\text { (mg/L as } \mathrm{P}) \\
(00671)\end{array}$ & $\begin{array}{c}\text { Coliform } \\
\text { fecal, } \\
0.7 \text { micron } \\
\text { membrane } \\
\text { filter } \\
(\text { cols } / 100 \mathrm{~mL} \text { ) } \\
(31625)\end{array}$ & $\begin{array}{c}\text { Strepto- } \\
\text { cocci } \\
\text { fecal, } \\
\text { KF agar } \\
\text { (cols } / 100 \mathrm{~mL} \text { ) } \\
(31673)\end{array}$ & $\begin{array}{c}\text { Well } \\
\text { owner }\end{array}$ \\
\hline$\overline{\mathrm{W} 1}$ & 0.01 & $<$ & & 0.07 & $<0.02$ & 0.01 & $<3$ & K33 & M. Bradley \\
\hline W2 & .01 & & & 2.6 & .03 & .03 & $\mathrm{~K} 3$ & $\mathrm{~K} 17$ & Q. Tipton \\
\hline W3 & .01 & & & $<.02$ & $<.02$ & .01 & $<3$ & $<4$ & Q. Tipton \\
\hline W4 & .01 & & & 6.0 & .03 & .02 & $\mathrm{~K} 15$ & $\mathrm{~K} 77$ & T. Benson \\
\hline W5 & .01 & & & 1.0 & .02 & .01 & K3 & $\mathrm{K} 17$ & K. Garrett \\
\hline Minimum & .01 & & & $<.02$ & $<.02$ & .01 & $<3$ & $<4$ & \\
\hline Maximum & .01 & & & 6.0 & .03 & .03 & $\mathrm{~K} 15$ & $\mathrm{~K} 77$ & \\
\hline Median & .01 & & & 1.0 & .02 & .01 & K3 & $\mathrm{K} 17$ & \\
\hline
\end{tabular}


Table 6.-Organic compound data for selected wells and springs in the Yocum Creek Basin $[\mu \mathrm{g} / \mathrm{L}$, micrograms per liter; five digit number in parentheses are STORET parameter codes used for computer storage of data; <, less than]

\begin{tabular}{|c|c|c|c|c|c|c|c|c|c|c|c|c|c|c|}
\hline \multirow{2}{*}{$\begin{array}{l}\text { Site ID } \\
\overline{\text { W1 }}\end{array}$} & \multirow{2}{*}{$\begin{array}{l}\begin{array}{l}\text { Date of } \\
\text { sample }\end{array} \\
7-27-93\end{array}$} & $\begin{array}{l}\text { Time of } \\
\text { sample }\end{array}$ & $\begin{array}{c}\text { Dichloro- } \\
\text { difluoro- } \\
\text { methane c } \\
(\mu \mathrm{g} / \mathrm{L}) \\
(34668)\end{array}$ & $\begin{array}{c}\text { Vinyl } \\
\text { chloride } \\
(\mu \mathrm{g} / \mathrm{L}) \\
(39175)\end{array}$ & \multicolumn{3}{|c|}{$\begin{array}{l}\text { Trichloro- } \\
\text { fluoro- } \\
\text { methane } \\
(\mu g / L) \\
(34488)\end{array}$} & \multicolumn{2}{|c|}{$\begin{array}{c}\text { 1,1-Dichloro } \\
\text { ethene } \\
(\mu \mathrm{g} / \mathrm{L}) \\
(34501)\end{array}$} & $\begin{array}{c}\text { Methylene } \\
\text { chloride } \\
(\mu \mathrm{g} / \mathrm{L}) \\
(34423)\end{array}$ & \multicolumn{2}{|c|}{$\begin{array}{l}\text { Trans-1,2- } \\
\text { dichloro- } \\
\text { ethane } \\
(\mu \mathrm{g} / \mathrm{L}) \\
(34546)\end{array}$} & $\begin{array}{c}\text { 1,1-Dichloro- } \\
\text { ethane } \\
(\mu \mathrm{g} / \mathrm{L}) \\
(34496)\end{array}$ & \multirow{2}{*}{$\begin{array}{c}\text { Cis } 1,2- \\
\text { Dichloroethene } \\
(\mu \mathrm{g} / \mathrm{L}) \\
(77093) \\
<0.2\end{array}$} \\
\hline & & 1700 & 0.2 & $<0.2$ & & 0.2 & & & 0.2 & $<0.2$ & $<0.2$ & & $<0.2$ & \\
\hline W5 & $7-29-93$ & 1500 & $<.2$ & $<.2$ & & $<.2$ & & & $<.2$ & $<.2$ & $<.2$ & & $<.2$ & $<.2$ \\
\hline SPR-3 & $7-28-93$ & 1040 & $<.2$ & $<.2$ & & $<.2$ & & & $<.2$ & $<.2$ & $<.2$ & & $<.2$ & $<.2$ \\
\hline SPR-13 & $8-02-93$ & 1615 & $<.2$ & $<.2$ & & $<.2$ & & & $<.2$ & $<.2$ & $<.2$ & & $<.2$ & $<.2$ \\
\hline Site ID & $\begin{array}{c}\text { Chloroform } \\
(\mu \mathrm{g} / \mathrm{L}) \\
(32106)\end{array}$ & $\begin{array}{r}1,1,1- \\
\text { chloroe } \\
\quad(\mu \mathrm{g} / \\
\quad(345\end{array}$ & $\begin{array}{lr} & \mathrm{Ca} \\
-\mathrm{Tri}- & \mathrm{te} \\
\text { ethane } & \mathrm{chl} \\
\mathrm{L}) & (\mu \\
06) & (32\end{array}$ & $\begin{array}{l}\text { Carbon } \\
\text { tetra- } \\
\text { hloride } \\
(\mu \mathrm{g} / \mathrm{L}) \\
32102)\end{array}$ & $\begin{array}{r}\text { Ben } \\
(\mu \mathrm{g} \\
(340\end{array}$ & $\begin{array}{l}\text { zene } \\
\text { (L) } \\
030)\end{array}$ & & $\begin{array}{l}\text { 2-Di- } \\
\text { loro- } \\
\text { hane } \\
\text { (g/L) } \\
\text { 2103) }\end{array}$ & $\begin{array}{r}\text { Trich } \\
\text { ethe } \\
(\mu \mathrm{g}) \\
(391\end{array}$ & $\begin{array}{lr}\text { loro- } & 1,2-\mathrm{Di} \\
\text { ene } & \text { prop } \\
/ \mathrm{L}) & (\mu \mathrm{g} \\
80) & (34\end{array}$ & $\begin{array}{l}\text { ichloro- } \\
\text { pane } \\
\text { g/L) } \\
541)\end{array}$ & $\begin{array}{r}\text { Bromodi } \\
\text { chloro- } \\
\text { methane } \\
(\mu \mathrm{g} / \mathrm{L}) \\
(32101)\end{array}$ & 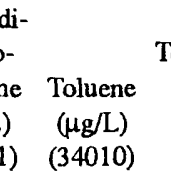 & $\begin{array}{c}\text { Tetrachloro- } \\
\text { ethene } \\
(\mu \mathrm{g} / \mathrm{L}) \\
(34475)\end{array}$ \\
\hline$\overline{\mathrm{W} 1}$ & $<0.2$ & $<0$. & $<0$. & 0.2 & $<0.2$ & & $<0.2$ & & $<0.2$ & & $\overline{0.2}$ & $<0.2$ & $<0.2$ & $<0.2$ \\
\hline W5 & $<.2$ & $<$ & $<$ & $<.2$ & $<.2$ & & $<.2$ & & $<.2$ & & $<.2$ & $<.2$ & $<.2$ & $<.2$ \\
\hline SPR-3 & $<.2$ & $<$ & $<$ & $<.2$ & $<.2$ & & $<.2$ & & $<.2$ & & $<.2$ & $<.2$ & $<.2$ & $<.2$ \\
\hline SPR-13 & .2 & $<$ & $<$ & $<.2$ & $<.2$ & & $<.2$ & & $<.2$ & & $<.2$ & $<.2$ & $<.2$ & $<.2$ \\
\hline Site ID & $\begin{array}{c}\text { Dibromo- } \\
\text { chloro- } \\
\text { methane } \\
(\mu \mathrm{g} / \mathrm{L}) \\
(32105)\end{array}$ & $\begin{array}{c}\text { Chloro- } \\
\text { benzene } \\
(\mu \mathrm{g} / \mathrm{L}) \\
(34301)\end{array}$ & $\begin{array}{c}\text { Ethyl- } \\
\text { benzene } \\
(\mu \mathrm{g} / \mathrm{L}) \\
(34371)\end{array}$ & $\begin{array}{r}\text { Dimet } \\
\text { benze } \\
\text { Xyler } \\
\text { tota } \\
(\mu \mathrm{g} / \\
(8155\end{array}$ & & $\begin{array}{l}\text { Styr } \\
(\mu \mathrm{g} \\
(771\end{array}$ & $\begin{array}{l}\text { rene } \\
3 / \mathrm{L}) \\
128)\end{array}$ & $\begin{array}{l}\text { Bro } \\
\text { for } \\
(\mu \mathrm{g} \\
(321\end{array}$ & $\begin{array}{l}\text { mo- } \\
\text { g/L) } \\
104)\end{array}$ & $\begin{array}{c}\text { 1,3-Dichloro } \\
\text { benzene } \\
\text { (meta) } \\
(\mu \mathrm{g} / \mathrm{L}) \\
(34566)\end{array}$ & $\begin{array}{r}1,4-\mathrm{D} \\
\text { ber } \\
(\mathrm{p} \\
(\mu \\
(3\end{array}$ & $\begin{array}{l}\text { Dichloro- } \\
\text { enzene } \\
\text { (para) } \\
(\mu \mathrm{g} / \mathrm{L}) \\
\mathbf{3 4 5 7 1 )}\end{array}$ & $\begin{array}{c}\text { 1,2-Dichloro- } \\
\text { benzene } \\
\text { (ortho) } \\
(\mu \mathrm{g} / \mathrm{L}) \\
(34536)\end{array}$ & $\begin{array}{c}\text { Trichloro- } \\
\text { trifluoro- } \\
\text { o- ethane } \\
(1,1,2-\mathrm{Cl} \\
1,2,2 \mathrm{~F}) \\
(\mu \mathrm{g} / \mathrm{L}) \\
(77652)\end{array}$ \\
\hline$\overline{\mathrm{W} 1}$ & $<0.2$ & $<0.2$ & $<0.2$ & $<0.2$ & & $<0.2$ & & $<0.2$ & & $<0.2$ & & $<0.2$ & $<0.2$ & $<0.5$ \\
\hline W5 & $<.2$ & $<.2$ & $<.2$ & $<.2$ & & $<.2$ & & $<.2$ & & $<.2$ & & $<.2$ & $<.2$ & $<.5$ \\
\hline SPR-3 & $<.2$ & $<.2$ & $<.2$ & $<.2$ & & $<.2$ & & $<.2$ & & $<.2$ & & $<.2$ & $<.2$ & $<.5$ \\
\hline SPR-13 & $<.2$ & $<.2$ & $<.2$ & $<.2$ & & $<.2$ & & $<.2$ & & $<.2$ & & $<.2$ & $<.2$ & $<.5$ \\
\hline
\end{tabular}


Table 7.--Discharge and water-quality data for springs located in the Yocum Creek Basin

[Temperature reported to nearest 0.5 degrees Celsius; ${ }^{\circ} \mathrm{C}$, degrees Celsius; five digit numbers in parentheses are STORET parameter codes used for computer storage of data; $\mathrm{mm}$ of $\mathrm{Hg}$, millimeters of mercury; $\mathrm{ft}^{3} / \mathrm{s}$, cubic feet per second; --, no data; $\mu \mathrm{S} / \mathrm{cm}$ at $25^{\circ} \mathrm{C}$, microsiemens per centimeter at 25 degrees Celsius; mg/L, milligrams per liter; WH, whole water; FET, fixed endpoint titration; $F$, field; $<$, less than; cols/100 $\mathrm{mL}$, number of colonies per 100 milliliters of sample; $\mathrm{K}$, non-ideal count; >, greater than]

\begin{tabular}{|c|c|c|c|c|c|c|c|c|c|c|}
\hline Site ID & $\begin{array}{l}\text { Date of } \\
\text { sample }\end{array}$ & $\begin{array}{c}\text { Time } \\
\text { of } \\
\text { sample }\end{array}$ & $\begin{array}{c}\text { Water } \\
\text { temper } \\
\text { ature } \\
\left({ }^{\circ} \mathrm{C}\right) \\
(00010)\end{array}$ & $\begin{array}{c}\text { Air } \\
\text { pressure } \\
\text { (mm of } \\
\mathrm{Hg}) \\
(00025)\end{array}$ & $\begin{array}{l}\text { Discharge, } \\
\text { instan- } \\
\text { taneous } \\
\left(\mathrm{ft}^{3} / \mathrm{s}\right) \\
(00061)\end{array}$ & $\begin{array}{c}\text { Specific } \\
\text { conductance } \\
(\mu \mathrm{S} / \mathrm{cm} \text { at } \\
\left.25^{\circ} \mathrm{C}\right) \\
(00095)\end{array}$ & $\begin{array}{l}\text { Oxygen, } \\
\text { dissolved } \\
(\mathrm{mg} / \mathrm{L}) \\
(00300)\end{array}$ & $\begin{array}{c}\mathrm{pH}, \\
\text { field } \\
\text { (standard } \\
\text { units) } \\
(00400)\end{array}$ & $\begin{array}{l}\text { Alkalinity, } \\
\text { WH, FET, F } \\
(\mathrm{mg} / \mathrm{L} \text { as } \\
\left.\mathrm{CaCO}_{3}\right) \\
(00410)\end{array}$ & $\begin{array}{c}\text { Nitrogen } \\
\text { ammonia, } \\
\text { dissolved } \\
(\mathrm{mg} / \mathrm{L} \text { as } \mathrm{N}) \\
(00608)\end{array}$ \\
\hline SPR-1 & $7-27-93$ & 1430 & 19.0 & 743 & 0.22 & 315 & 8.2 & 8.0 & 138 & 0.01 \\
\hline SPR-2 & $7-28-93$ & 1020 & 17.0 & 737 & .67 & 298 & 6.4 & 7.1 & 136 & .02 \\
\hline SPR-3 & $7-28-93$ & 1040 & 16.0 & 736 & 6.6 & 288 & 6.2 & 7.0 & 128 & .01 \\
\hline SPR-4 & $7-28-93$ & 1000 & 14.0 & 737 & .58 & 394 & 8.3 & 7.4 & 190 & .01 \\
\hline SPR-5 & $7-28-93$ & 1400 & 17.5 & 733 & 2.3 & 329 & 7.5 & 7.4 & 140 & .01 \\
\hline SPR-8 & $7-29-93$ & 0910 & 14.5 & 737 & 1.4 & 410 & 7.8 & 7.2 & 200 & .01 \\
\hline SPR-9 & $7-29-93$ & 1610 & 14.5 & 732 & .21 & 369 & 4.0 & 6.8 & 152 & .01 \\
\hline SPR-10 & $8-02-93$ & 1245 & 14.0 & 732 & 5.6 & 333 & 6.9 & 6.8 & 148 & .03 \\
\hline SPR-11 & $8-02-93$ & 1420 & 15.0 & 732 & - & 436 & 7.2 & 7.5 & 184 & .03 \\
\hline SPR-12 & $7-29-93$ & 1400 & 20.5 & 732 & 1.1 & 441 & 9.9 & 7.6 & 178 & .02 \\
\hline SPR-13 & $8-02-93$ & 1615 & 16.0 & 732 & 3.7 & 460 & 7.1 & 6.8 & 184 & .03 \\
\hline SPR-14 & $8-02-93$ & 1550 & 20.0 & 732 & .51 & 518 & 7.1 & 7.9 & 256 & .04 \\
\hline Minimum & & & 14.0 & & .21 & 288 & 4.0 & 6.8 & 128 & .01 \\
\hline Maximum & & & 20.5 & & 6.6 & 518 & 9.9 & 8.0 & 256 & .04 \\
\hline Median & & & 15.5 & & 1.1 & 382 & 7.2 & 7.3 & 165 & .02 \\
\hline Site ID & $\begin{array}{c}\text { Nitrogen, } \\
\text { nitrite } \\
\text { dissolved } \\
(\mathrm{mg} / \mathrm{L} \text { as } \mathrm{N}) \\
(00613)\end{array}$ & $\begin{array}{r}\mathrm{Ni} \\
\mathrm{amm} \\
\text { or } \\
\mathrm{dis} \\
(\mathrm{mg} \\
(0\end{array}$ & $\begin{array}{l}\text { rogen, } \\
\text { onia plus } \\
\text { ganic } \\
\text { solved } \\
\text { L as N) } \\
0623 \text { ) }\end{array}$ & $\begin{array}{l}\text { Nitrogen, } \\
\text { nitrite plus } \\
\text { nitrate, } \\
\text { dissolved } \\
(\mathrm{mg} / \mathrm{L} \text { as } \mathrm{N}) \\
(00631)\end{array}$ & $\begin{array}{l}\text { Phos- } \\
\text { phorus, } \\
\text { total } \\
(\mathrm{mg} / \mathrm{L} \text { as } \mathrm{P}) \\
(00665)\end{array}$ & $\begin{array}{c}\text { Phos- } \\
\text { phorus } \\
\text { ortho, } \\
\text { dissolved } \\
\text { (mg/L as } \mathrm{P}) \\
(00671)\end{array}$ & $\begin{array}{c}\text { Coliform } \\
\text { fecal, } \\
0.7 \text { micron } \\
\text { membrane } \\
\text { filter } \\
\text { (cols/ } \\
100 \mathrm{~mL}) \\
(31625)\end{array}$ & $\begin{array}{r}\text { Strep } \\
\text { coc } \\
\text { feca } \\
\mathrm{KF} \mathrm{a} \\
(\mathrm{col} \\
100 \mathrm{~m} \\
(316\end{array}$ & & Name \\
\hline$\overline{\text { SPR-1 }}$ & 0.01 & & 0.20 & 2.0 & 0.02 & 0.02 & $\mathrm{~K} 12$ & 280 & \multicolumn{2}{|c|}{ Bear Hollow Spring } \\
\hline SPR-2 & .01 & & .49 & 1.9 & .02 & .01 & K6 & 120 & \multicolumn{2}{|c|}{--} \\
\hline SPR-3 & .01 & & .20 & 1.9 & .03 & .01 & K48 & 700 & \multicolumn{2}{|c|}{ Spring Creek Spring } \\
\hline SPR-4 & .01 & & .26 & 2.1 & .03 & .02 & K58 & $>2,000$ & \multicolumn{2}{|c|}{ Tipton Spring } \\
\hline SPR-5 & .01 & & $<.20$ & 1.4 & $<.02$ & .01 & $\mathrm{~K} 12$ & 110 & \multicolumn{2}{|c|}{ Stillhouse Spring } \\
\hline SPR-8 & .01 & & $<.20$ & 2.6 & .02 & .01 & $\mathrm{~K} 33$ & $\mathrm{~K} 420$ & \multicolumn{2}{|c|}{ Tipton Spring } \\
\hline SPR-9 & .01 & & $<.20$ & 3.6 & .03 & .03 & K200 & 400 & \multicolumn{2}{|c|}{ Duncan Spring } \\
\hline SPR-10 & $<.01$ & & $<.20$ & 1.8 & .03 & .03 & $\mathrm{~K} 3$ & 160 & \multicolumn{2}{|c|}{ Anderson Spring } \\
\hline SPR-11 & $<.01$ & & .20 & 7.0 & .06 & .07 & $\mathrm{~K} 21$ & 240 & \multicolumn{2}{|c|}{ Garrett Spring } \\
\hline SPR-12 & .01 & & $<.20$ & 2.5 & .08 & .07 & 80 & $>2,000$ & \multicolumn{2}{|l|}{-} \\
\hline SPR-13 & $<.01$ & & $<.20$ & 4.2 & .08 & .06 & $\mathrm{~K} 15$ & 170 & \multicolumn{2}{|c|}{ Newhome Spring } \\
\hline SPR-14 & $<.01$ & & .24 & 2.1 & .07 & .02 & K6 & 980 & \multicolumn{2}{|c|}{--} \\
\hline Minimum & $<.01$ & & $<.20$ & 1.4 & $<.02$ & .01 & $\mathrm{~K} 3$ & 110 & \multicolumn{2}{|l|}{--} \\
\hline Maximum & .01 & & .49 & 7.0 & .08 & .07 & $\mathrm{~K} 200$ & $>2,000$ & \multicolumn{2}{|l|}{-. } \\
\hline Median & .01 & & $<.20$ & 2.1 & .03 & .02 & 18 & 350 & \multicolumn{2}{|l|}{--} \\
\hline
\end{tabular}




\section{STREAMFLOW GAIN AND LOSS}

Numerous discharge measurements were made in the Yocum Creek Basin to determine the connection between the stream and the underlying Springfield aquifer. Three losing streamflow reaches were identified during the study of the Yocum Creek Basin (fig. 2). The mainstem of Yocum Creek was characterized by one losing reach. A discharge of $3.7 \mathrm{ft}^{3} / \mathrm{s}$ was measured at SPR-13 (Newhome Spring). Surface flow began to decrease approximately 75 $y$ ds from the orifice of the spring, and two-tenths of a mile from SPR-13 all surface flow was redirected to subsurface flow. The mainstem of the creekbed was dry for a distance of approximately four-tenths of a mile, where $1.1 \mathrm{ft}^{3} / \mathrm{s}$ of surface flow was measured at SPR-12.

Two losing reaches were located on tributaries feeding Yocum Creek. Trib-5, located on Stillhouse Creek, is four-tenths of a mile from the confluence of Yocum Creek. Surface flow was measured at $2.6 \mathrm{ft}^{3} / \mathrm{s}$. Surface flow decreased steadily until the creekbed was dry for a distance of two-tenths of a mile from the confluence of Yocum Creek. The other losing tributary was located on Braswell Branch. A discharge of $0.51 \mathrm{ft}^{3} / \mathrm{s}$ was measured at SPR-14. Surface flow disappeared one-tenth of a mile downstream from SPR-14. Surface flow did not reoccur for approximately $1.6 \mathrm{mi}$ at SPR-13.

Large producing springs (SPR-13, SPR-12, and SPR-10) located in the mid to upper reaches of the basin contribute most of the flow to Yocum Creek. Streamflow increased an average of 29 percent in each of the 11 gaining reaches of the mainstem from site YM-12 to YM-1 at the mouth. The largest increase occurred between sites YM-11 and YM-10 where a 140 percent increase in surface flow was measured. These observations suggest that significant interaction exists between the underlying Springfield aquifer and surface flow in the Yocum Creek Basin.

\section{SUMMARY}

A study of the Yocum Creek Basin was undertaken between July 27 and August 3, 1993, to describe the current water-quality conditions of the surface and ground water in the basin. Physical, nutrient, bacteriological, common constituent, selected metal, and volatile organic compound data were collected at various sites in the basin. Streamflow measurements were conducted at various locations along the mainstem and at sites on major tributaries to identify gaining and losing reaches of streamflow.

Dissolved nitrite plus nitrate concentrations ranged from 1.3 to $3.8 \mathrm{mg} / \mathrm{L}$ as nitrogen in surface-water samples. A ground-water sample (W-4), contained a dissolved nitrite plus nitrate concentration of $6.0 \mathrm{mg} / \mathrm{L}$ as nitrogen, which is approaching the U.S. Environmental Protection Agency maximum contaminant level for drinking water of 10 $\mathrm{mg} / \mathrm{L}$ as nitrogen (U.S. Environmental Protection Agency, 1993). Dissolved nitrite plus nitrate concentrations ranged from 1.4 to $7.0 \mathrm{mg} / \mathrm{L}$ as nitrogen in springs sampled.

Fecal coliform bacteria counts for surface-water sites ranged from 9 to $220 \mathrm{cols} / 100 \mathrm{~mL}$ with a median of 49 cols $/ 100 \mathrm{~mL}$. Fecal streptococci bacteria counts for surface-water sites ranged from 37 to $1,500 \mathrm{cols} / 100 \mathrm{~mL}$ with a median of $420 \mathrm{cols} / 100 \mathrm{~mL}$. Fecal coliform bacteria counts for springs ranged from 3 to $200 \mathrm{cols} / 100 \mathrm{~mL}$ with a median of $18 \mathrm{cols} / 100 \mathrm{~mL}$. Fecal streptococci bacteria counts for springs ranged from 110 to more than 2,000 cols $/ 100 \mathrm{~mL}$ with a median of $350 \mathrm{cols} / 100 \mathrm{~mL}$.

Analyses for selected metals at one mainstem site, two springs, and two well sites indicate that metals were not present in significant concentrations. Volatile organic compound samples were collected at two wells and two springs. Chloroform was the only volatile organic compound found to be above the detection limit. Analysis indicated that 0.2 $\mu \mathrm{g} / \mathrm{L}$ of chloroform were present in SPR-13.

Diel dissolved oxygen concentrations and temperatures were measured at an upstream and downstream site on the mainstem of the stream. At the upstream site (YM-8), dissolved oxygen concentrations ranged from 6.2 to 9.9 $\mathrm{mg} / \mathrm{L}$ and temperatures ranged from 18.5 to $23.0^{\circ} \mathrm{C}$. Dissolved oxygen concentrations were higher and temperature values were lower at the upstream site than those at the downstream site.

Large producing springs located in the mid to upper reaches of the basin contribute most of the flow to Yocum Creek. Streamflow increased an average of 29 percent on the mainstem of the stream. One losing reach was discovered on the mainstem of the stream and two losing reaches on tributaries to the mainstem. Surface flow steadily decreased along these reaches to the point where surface flow was not present, and the streambed became dry. These observations suggest that significant interaction exists between the underlying Springfield aquifer and surface flow in the Yocum Creek Basin. 


\section{REFERENCES}

Britton, L.J., and Greeson, P.E., eds., 1987, Methods for collection and analysis of aquatic biological and microbiological samples: U.S. Geological Survey Techniques of Water-Resources Investigations, book 5, chap. A4, p. 37-50.

Buchanan, T.J., and Sommers, W.P., 1984, Discharge measurements at gaging stations: U.S. Geological Survey Techniques of Water-Resources Investigations, book 3, chap. A8, 65 p.

Caplan, W.M., 1960, Subsurface geology of pre-Everton rocks in northern Arkansas: Arkansas Geological and Conservation Commission Information Circular 21, $17 \mathrm{p}$.

Croneis, C.C., 1930, Geology of Arkansas Paleozoic area: Arkansas Geological Survey Annual Report, v. 4, 457 p.

Fenneman, N.M., 1938, Physiography of eastern United States: New York, McGraw-Hill Book Co., Inc., 714 p.

Fowlkes, D.H., Harper, M.D., and McCright, R.T., 1981, Soil survey of Carroll County, Arkansas: U.S. Soil Conservation Service, $119 \mathrm{p}$.

Frezon, S.E., and Glick, E.E., 1959, Pre-Atoka rocks of Arkansas: U.S. Geological Survey Professional Paper 314-H, p. 171-189.

Lamonds, A.G., 1972, Water-resources reconnaissance of the Ozarks Plateaus Province, northern Arkansas: U.S. Geological Survey Hydrologic Investigations Atlas 383, 2 sheets.

Sullavan, J.N., 1974, Drainage areas of streams in Arkansas-White River Basin: U.S. Geological Survey Open-File Report, 123 p.

U.S. Department of Commerce, National Oceanic and Atmospheric Administration, 1992, Climatological data annual summary (by state): Asheville, North Carolina, National Climatic Data Center.

U.S. Environmental Protection Agency, 1994, Drinking water regulations and health advisories: EPA 822-R-94-001, $11 \mathrm{p}$.

Ward, J.R., and Harr, C.A., eds., 1990, Methods for collection and processing of surface-water and bed-material samples for physical and chemical analysis: U.S. Geological Survey Open-File Report 90-140, 71 p. 